$K(x, y)=\left\{\begin{array}{l}f_{s}(y) \text { if } x^{1}=s<1 \text { and } \int_{0}^{\eta} f_{s}<\infty, \\ \prod_{j=1}^{m} 1 / x^{j} \text { if } x^{1} \geqq 1 \text { and } 0<y^{j}<x^{j} \text { for all } j, \\ 0 \text { otherwise. }\end{array}\right.$

Then $K$ is regular on $\mathfrak{B}_{m}$; so $\mathfrak{\Omega} \mathfrak{B}_{m}$. If $f \boldsymbol{\varepsilon} \mathfrak{B}_{m}-\mathfrak{B}_{m}$, either there is a $b>0$ such that $B$ is of finite measure and $\int_{B} f$ exists or not; if there is no such $b,|f|$ dominates a function with this property. Hence there is a function $f_{0}(y)$, zero if $y$ is not in $B$, such that $\int_{B} f_{0}$ exists and $\int_{B} f_{0} f$ does not. Then there is an $s<1$ such that $f_{s}$ dominates $f_{0}$ but $\int_{0}^{\eta} f_{s}<\infty$. Hence there is an $x$ such that $U_{x}(f)$ does not exist; so $\mathfrak{B}_{m} \supset \Omega$.

For $m \geqq 2$ this construction can be modified to give a kernel with $\Re_{m}$ as domain of regularity.

BROWN UNIVERSITY

\title{
BOUNDED SELF-ADJOINT OPERATORS AND THE PROBLEM OF MOMENTS*
}

\section{B. A. LENGYEL}

It is known that there exists a close connection between the theory of moments and Jacobi matrices on one side, and the theory of selfadjoint operators in Hilbert space on the other. This connection has been thoroughly investigated by Stone in the tenth chapter of his textbook on Hilbert space. $\dagger$ The solution of both the moment problem and the spectral resolution of self-adjoint operators relies on the possibility of representing a class of analytic functions with positive imaginary parts in the upper half-plane by Stieltjes integrals of the form

$$
\int_{-\infty}^{+\infty} \frac{d \rho(\lambda)}{\lambda-z}
$$

However, the spectral resolution requires the representation of more general functions than those involved in the solution of the problem of moments. The bounded self-adjoint operators do not, and I wish to show that the spectral theorem for bounded operators can be deduced immediately from the well known theorems concerning the problem of moments. Let $H$ be a bounded self-adjoint operator, $f$ an element of the Hilbert space, $R_{2}=(H-z I)^{-1}$ the resolvent of $H$. It

* Presented to the Society, September 6, 1938.

$\dagger$ M. H. Stone, Linear Transformations in Hilbert Space and their Applications to Analysis, American Mathematical Society Colloquium Publications, vol. 15, New York, 1932. The notations of this textbook are used throughout the present paper. 
is known that in order to prove the standard form of the spectral theorem it is sufficient to prove that given any $f$ there exists a unique monotone increasing function $\rho(\lambda)$ of bounded variation, such that

$$
\left(R_{z} f, f\right)=\int_{-\infty}^{+\infty} \frac{d \rho(\lambda)}{\lambda-z}
$$

for all values of $z{ }^{*}$ The resolvent of a bounded self-adjoint operator can be developed in a series:

$$
-R_{z}=\frac{I}{z}+\frac{H}{z^{2}}+\frac{H^{2}}{z^{3}}+\cdots,
$$

or

$$
-\left(R_{z} f, f\right)=\frac{c_{0}}{z}+\frac{c_{1}}{z^{2}}+\frac{c_{2}}{z^{3}}+\cdots,
$$

where $c_{i}=\left(H^{i} f, f\right)$, and the series is convergent for sufficiently large values of $z$.

Now, if the moment problem has a solution for the sequence $\left\{c_{i}\right\}$; that is, if there exists a $\rho(\lambda)$ such that

$$
c_{i}=\int_{-\infty}^{+\infty} \lambda^{i} d \rho(\lambda)
$$

for all $i$, then

$$
\left(R_{z} f, f\right)=-\sum_{i=0}^{\infty} \int_{-\infty}^{+\infty} \frac{\lambda^{i}}{z^{i+1}} d \rho(\lambda)=\int_{-\infty}^{+\infty} \frac{d \rho(\lambda)}{\lambda-z},
$$

and the spectral problem is solved.

Let us consider the quadratic forms

$$
\left|\sum_{i=0}^{n} x_{i} H^{i} f\right|^{2}=\sum_{i, j=0}^{n} x_{i} \bar{x}_{j}\left(H^{i} f, H^{j} f\right)=\sum_{i, j=0}^{n} c_{i+j} x_{i} \bar{x}_{i}, \quad n=1,2, \cdots .
$$

These forms are all definite positive for every $f$ and $n$; therefore their determinants

$$
c_{n}=\left|\begin{array}{llll}
c_{0} & c_{1} & \cdots & c_{n} \\
c_{1} & c_{2} & \cdots & c_{n+1} \\
\cdot & \cdot & \cdots & . \\
c_{n} & c_{n+1} & \cdots & c_{2 n}
\end{array}\right|
$$

* Cf. B. O. Koopman and J. L. Doob, this Bulletin, vol. 40 (1934), pp. 601-605, or B. A. Lengvel. Acta Scientiarum Mathematicarum. Szeged, vol. 9 (1939). 
are all positive. This is the well known condition for the existence of a solution of the problem of moments.

In order to prove the uniqueness of the solution we make use of a theorem of Hamburger.* Let $M_{n}$ be the minimum of the definite quadratic form

$$
\sum_{i, j=0}^{n} c_{i+j} x_{i} \bar{x}_{j}
$$

for $x_{0}=1$, and let $N_{n}$ be the minimum of

$$
\sum_{i, j=0}^{n} c_{i+j+2} x_{i} \bar{x}_{j}
$$

for $x_{0}=1$. Evidently both $M_{n}$ and $N_{n}$ are nonnegative; furthermore $M_{n} \geqq M_{n+1}, N_{n} \geqq N_{n+1}$; therefore, $M=\lim M_{n}$ and $N=\lim N_{n}$ exist.

The necessary and sufficient condition for the uniqueness of the solution of the moment problem is that at least one of the numbers $M$ and $N$ is 0 .

Thus, to prove the uniqueness of $\rho(\lambda)$ we have to show that for every $f$, at least one of the sequences

$$
M_{n}=\min \left|f-\sum_{i=1}^{n} x_{i} H^{i} f\right|^{2}, \quad N_{n}=\min \left|H f-\sum_{i=1}^{n} x_{i} H^{i+1} f\right|^{2}
$$

tends to 0 . This means that $f$ can be approximated by linear combinations of $H f, H^{2} f, \cdots$; or $H f$ can be approximated by linear combinations of $H^{2} f, H^{3} f, \cdots$; or both approximations are possible. In other words, if $\mathfrak{M}_{f}$ is the closed linear manifold determined by $H f, H^{2} f, \cdots$, then at least one of the following inclusion relations holds:

$$
\begin{gathered}
f \varepsilon \mathfrak{M}_{f}, \\
H f \varepsilon \mathfrak{M}_{H f} .
\end{gathered}
$$

This lemma is interesting in itself; its proof can be carried out as follows.

Assume that $\mathfrak{M}_{n}$ is the closed linear manifold determined by $\left[H f, H^{2} f, \cdots, H^{n} f\right]$. Evidently $\mathfrak{M}_{n} \subset \mathfrak{M}_{n+1}$ and $H \mathfrak{M}_{n} \subset \mathfrak{M}_{n+1}$. Therefore, $\mathfrak{M}_{f}=\lim _{n \rightarrow \infty} \mathfrak{M}_{n}$ exists and $H \mathfrak{M}_{f} \subset \mathfrak{M}_{f}$. The element $f$ can be re-

${ }^{*}$ H. Hamburger, Mathematische Annalen, vol. 81 (1920), p. 234. Hamburger uses the same quadratic forms with real variables. This evidently does not affect the minima. 
solved into the sum of $f_{1}$ and $f_{2}$, with $f_{1}$ in $\mathfrak{M}_{f}$ and $f_{2}$ in $\mathfrak{S}-\mathfrak{M}_{f}$, the orthogonal complement of $\mathfrak{M}_{f}$.

Either $f_{2}=0$, whence $f \varepsilon \mathfrak{M}_{f}$, or $f_{2} \neq 0$. In the latter case we apply $H^{2}$ to $f=f_{1}+f_{2}$ and multiply by $f_{2}$. This gives

$$
\left(H^{2} f, f_{2}\right)=\left(H^{2} f_{1}, f_{2}\right)+\left(H^{2} f_{2}, f_{2}\right) .
$$

Since $H^{2} f$ and $H^{2} f_{1}$ are in $\mathfrak{M}_{f}$, their scalar products with $f_{2}$ give 0 ; thus

$$
\left(H^{2} f_{2}, f_{2}\right)=\left|H f_{2}\right|^{2}=0 .
$$

The element $f_{1}$ is in the closed linear manifold $\mathfrak{M}_{f}$, so that for suitable $x_{1}^{(n)}, x_{2}^{(n)}, \cdots, x_{n}^{(n)}$ we have

$$
\left|f-f_{2}-\sum_{i=1}^{n} x_{i}^{(n)} H^{i f}\right|=\left|f_{1}-\sum_{i=1}^{n} x_{i}{ }^{(n)} H^{i f}\right|=\epsilon_{n},
$$

where $\epsilon_{n} \rightarrow 0$. Applying the bounded operator $H$ to

$$
f-f_{2}-\sum_{i=1}^{n} x_{i}^{(n)} H^{i f}
$$

and using (3), we obtain

$$
\left|H f-\sum_{i=1}^{n} x_{i}^{(n)} H^{i+1} f\right| \rightarrow 0, \quad \text { if } \quad n \rightarrow \infty ;
$$

that is, (2) holds. Thereby the proof of our statement is complete.

WORCESTER, Mass. 